\title{
A QoE Aware Fairness Bi-level Resource Allocation Algorithm for Multiple Video Streaming in WLAN
}

\author{
(Invited Paper)
}

\author{
Hu Zhou, Bo Li, Zhongjiang Yan, Xiaoya Zuo, Mao Yang \\ School of Electronics and Information \\ Northwestern Polytechnical University, Xi' an, China \\ Email: zhouhu@mail.nwpu.edu.cn \\ \{libo.npu, zhjyan, zuoxy, yangmao\}@nwpu.edu.cn
}

\begin{abstract}
With the increasing of smart devices such as mobile phones and tablets, the scenario of multiple video users watching video streaming simultaneously in one wireless local area network (WLAN) becomes more and more popular. However, the quality of experience (QoE) and the fairness among multiple users are seriously impacted by the limited bandwidth and shared resources of WLAN. In this paper, we propose a novel bi-level resource allocation algorithm. To maximize the total throughput of the network, the WLAN is firstly tuned to the optimal operation point. Then the wireless resource is carefully allocated at the first level, i.e., between AP and uplink background traffic users, and the second level, i.e., among downlink video users. The simulation results show that the proposed algorithm can guarantee the QoE and the fairness for all the video users, and there is little impact on the average throughput of the background traffic users.
\end{abstract}

Keywords-quality of the experience (QoE), fairness, resource allocation, HTTP video streaming, wireless local area network (WLAN).

\section{INTRODUCTION}

As one of the most popular services over the Internet, the video streaming shows a rising trend year by year. According to Cisco's report [1], the video traffic has reached 52 percent of mobile data traffic by the end of 2013 and will account for over 70 percent by 2019. With the rapid development of video websites (such as YouTube and YouKu), HTTP video streaming, which guarantees the transmission of video packets and reduces the complexity of video server, becomes one of the most important video streaming transmission technologies [2]. On the other hand, wireless local area network (WLAN) based on IEEE 802.11 standards has become one of the most popular wireless access approaches for its widespread deployment and low price (even free in most cases). Ericsson reports that the heavy and medium video users in the US and UK prefer watching the online video over WLAN rather than via cellular networks [3]. Moreover, due to the popularity of smart devices, such as mobile phones and tablets, it is more and more common that many users watch video streaming or upload/download data files (e.g., P2P sharing) in one WLAN and compete for the limited wireless resource collectively, especially in hotspots, such as airports and shopping malls.

Quality of experience (QoE), which is defined as the overall acceptability of an application or service perceived subjectively by the end users [4], is one of the most important factors determining the success of a video service. Actually, for the HTTP video streaming, both the frequency and the duration of the stalling events impact the QoE severely [5], [6]. If a video user experiences too many stalling events, he may get bored and even close the video. Meanwhile, in the multiple video streaming scenario, the fairness of QoE among video users also plays an important role on the success of the video service. However, both the QoE and the fairness are impacted by the features of WLAN, such as the uplink/downlink channel sharing mechanism, the contention based media access protocol, and the randomly backoff algorithm. Therefore, to guarantee the QoE for each HTTP video streaming and the fairness among multiple video users at the same time in WLAN becomes a significant and difficult problem. To the best knowledge of the authors, this problem has not been properly solved.

There are some studies focusing on the QoE performance of HTTP video streaming in WLAN, from the viewpoints of both the video streaming and the wireless network. The basic idea of the video streaming viewpoint is to adjust the bit rates of video chunks downloading from the video server according to the bandwidth obtained by video users, which is also known as the HTTP adaptive bit rate streaming. Choi et al. [7] adopt a statistical method to estimate the bandwidth obtained by video users, then the corresponding bit rates can be selected. In [8] video users adjust the bit rate of the next video chunk according to the buffer level in seconds of playback. Miller et al. [9] formulate the bit rates adaptation problem as a MultipleChoice Nested Knapsack Problem and calculate the optimal adaptation trajectories based on the complete information of the throughput process. However, since all the video users compete for the limited downlink throughput of the Access Point (AP), when the number of video users is large, even the reliable transmission of video chunks with minimum bit rate may not be guaranteed, which results in the stalling events and bad QoE performance. Moreover, the bit rate adjustment mechanism implemented by each video user individually leads to a greedy competition and unfairness among all the video users.

For the studies from the viewpoint of wireless network, Zhou et al. [10] propose an admission control and network parameter adjustment algorithm, where new users are admitted into the WLAN only if the QoE of video users and the throughput of background users can be guaranteed. However, in [10] only the constant bit rate (CBR) video streaming is 
considered and the QoE is guaranteed to an expectation value but not to avoid the stalling events. Moreover, in the IEEE 802.11 family, IEEE 802.11e [11] is proposed to provide QoS for video streaming with higher channel access priority. However, the simple access category queue with First In First Out (FIFO) characteristic and the constant MAC layer parameters configuration make it inflexible for the multiple video streaming scenario. IEEE 802.11aa [12] is standardized recently to provide efficient and robust transmission for voice and video traffic in WLAN, however it only focuses on the multicast traffic and is unsuitable for HTTP video streaming.

In this paper, we aim to guarantee the QoE and fairness of multiple HTTP video streaming in WLAN from the viewpoint of wireless network. In the considered WLAN scenario, two kinds of users are considered besides of the AP, i.e., the video users and background traffic users. Particularly, the background traffic users are assumed to compete for the uplink bandwidth, and all of the video users are assumed to share the downlink bandwidth of the AP. Thus there exists a tradeoff between the downlink and uplink bandwidth, and further there is a competition among the video users. Therefore, a novel QoE aware fairness bi-level resource allocation algorithm is proposed. Firstly, the WLAN is tuned to the optimal operation point to maximize the total throughput of the network. Then the wireless channel resource is allocated at the first level, i.e., between AP and the background traffic users. Next, the wireless resource is allocated at the second level, i.e., among the downlink video users. The contributions of this paper can be summarized as follows:

- The resource allocation between the AP and background traffic users is achieved by properly adjusting the minimum contention windows of them, which guarantees the download rates of all the video users and avoids the occurrences of stalling events.

- The priorities of video users are calculated with a token-counter mechanism, and according to that the resource allocation among the video users is performed to guarantee the fairness.

- Extensive simulations to verify the performance of our algorithm are carried out, and simulation results prove that our algorithm outperforms IEEE 802.11e and IEEE DCF with Round-Robin in terms of both QoE and fairness, especially when the number of background traffic users is large.

The rest of this paper is organized as follows. In Section II, the system model is introduced in detail. Our bi-level resource allocation algorithm is presented in Section III. The performance evaluation by simulation is given in Section IV. Finally, Section V concludes the paper.

\section{SYSTEM MODEL}

\section{A. Network scenario}

We consider the WLAN scenario as shown in Fig.1. The $\mathrm{AP}$ is connected to the video server through the Internet. Excluding the AP, there are two kinds of users in the network, which are video users and background traffic users. For simplicity, the downlink background traffic is not considered in our scenario, but our algorithm is still effective when there

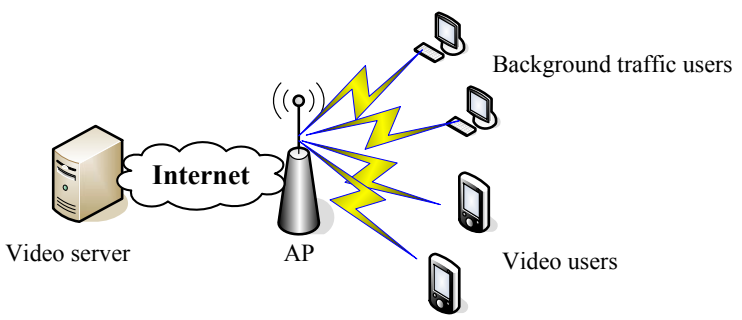

Fig. 1. Network scenario.

are downlink background traffic users in the WLAN. Each video user requires one HTTP progressive download video streaming service from the video server. Background traffic users send saturated background traffic to the AP by using the User Datagram Protocol (UDP). The length of packets sent by both AP and background traffic users is the same with the maximum transmission unit (MTU) of the MAC layer. AP serves each video user with one separate queue in its MAC layer, and allocates the transmission resource among those queues by a scheduler. Since we mainly focus on the characteristics of video streaming over WLAN, the transmission over wired network is assumed to be ideal, and the queues of AP are assumed to be backlogged with video data before the video streaming sessions are finished. Moreover, the impact of acknowledgement packets from transport layer sent by video users is ignored, as their length is much smaller than that of video packets.

\section{B. HTTP video streaming and QoE metric}

The HTTP video streaming session consists of two stages, i.e., the initial stage and the watching stage. In the initial stage, the video user downloads video data with a duration of $T_{b s}$ into the playback buffer to reduce the influence of fluctuation of bandwidth. In the watching stage, the playback starts and the video data are continuously downloaded. If the playback buffer goes empty, the playback stalls and the playback buffer is needed to be refilled to $T_{b s}$ before the playback can resume. We have studied the impact of the stalling events on the QoE of HTTP video streaming and proposed an objective assessment metric in [10], named as playback fluency $F$, which is defined as

$$
F \triangleq \frac{T_{v}}{T_{w}}=\frac{T_{v}}{T_{b s}+T_{p}},
$$

where $T_{v}$ is the actual playback duration of the target video streaming and is considerably larger than $T_{b s}$ in most cases, $T_{w}$ is the duration of the whole video streaming session, and $T_{p}$ is the sum of the actual playback duration and total length of stalling events. The QoE metric $F$ is a value in the range of 0 to 1 , and the more times of stalling events occurred and the longer of each stalling events, the smaller of $F$. If the stalling events are so rare that they can be almost eliminated, $F$ approaches to 1 , which indicates a satisfied QoE for video users.

\section{Our target}

In this paper, our target is to guarantee the QoE and fairness of multiple HTTP video streaming in WLAN. In particular, the QoE is represented by the average value of playback fluency $F$ of all the video streaming sessions and the fairness is denoted 
by the standard deviation of playback fluency $F$ of all the video streaming sessions. We try to eliminate the stalling events during the watching stage of each video streaming to make $F$ close to 1 and to properly allocate channel resources among multiple video users to reduce the standard deviation of $F$.

Next, a necessary condition to avoid the stalling events is given in an average sense. Assume that there are $n$ video users in the WLAN, and the watching stages of them start at the same time $t$. We focus on the performance of stalling events at time $t+\Delta t$, where $\Delta t$ is an arbitrary positive value. Let $v_{i}^{\prime}$ and $r_{i}^{\prime}$ denote the average video bit rate and the average download rate (in application layer, similarly hereinafter) of video user $i$ during $\Delta t$, respectively. In order to avoid the stalling events, the playback time of video data in the playback buffer should be larger than zero, thus we have

$$
T_{b s}+\left(r_{i}^{\prime} \Delta t-v_{i}^{\prime} \Delta t\right) / v_{i}^{\prime}>0, \quad 1 \leq i \leq n .
$$

Therefore the average download rate should meet that

$$
r_{i}^{\prime}>v_{i}^{\prime}\left(1-T_{b s} / \Delta t\right), \quad 1 \leq i \leq n
$$

In the multiple video streaming scenario, the necessary condition of avoiding stalling events for all the video streaming sessions is given as

$$
\sum_{i=1}^{n} r_{i}^{\prime}>\sum_{i=1}^{n} v_{i}^{\prime}\left(1-T_{b s} / \Delta t\right)
$$

Moreover, the duration of video streaming is considerably larger than $T_{b s}$ in most cases, then we consider that when $\Delta t \gg T_{b s}$, (3) can be derived as

$$
r_{i}^{\prime}>v_{i}^{\prime}, \quad 1 \leq i \leq n .
$$

and (4) can be given as

$$
\sum_{i=1}^{n} r_{i}^{\prime}>\sum_{i=1}^{n} v_{i}^{\prime}
$$

Therefore, it can be concluded that in order to avoid the stalling events for all the video streaming sessions during the whole watching stage, the sum of average download rates of all the video users should be larger than the sum of video bit rates.

\section{Resource allocation problems}

In order to guarantee the QoE and fairness of video streaming in WLAN, both the resource allocation between AP (downlink traffic) and background traffic users (uplink traffic) and the resource allocation among the video users should be considered carefully. Usually, the former is performed by the adjustment of the channel access probability of AP and background traffic users, and the latter is achieved by the scheduling algorithm of AP.

On one hand, since the download throughput of AP $r_{a p}$ is shared by all the video users, i.e., $r_{a p}=\sum_{i=1}^{n} r_{i}^{\prime}$, in order to meet the necessary condition in (6), the throughput of AP should be allocated dynamically according to the sum of required download rates of all the video users. On the other hand, due to the variable bit rate nature, different video users have different video bit rate requirements at different times. If the resource allocation among the video users does not consider the variability of video bit rates, e.g., only adopting the Round-Robin algorithm, some video playback buffers may go empty, which results in stalling event, while some other video playback buffers accumulate too much video data, which results in storage pressure. Therefore, from the viewpoint of wireless network, the bi-level resource allocation to guarantee the QoE and fairness of multiple HTTP video streaming in WLAN should be considered.

\section{TWO LEVEL RESOURCE ALLOCATION ALGORITHM}

The basic idea of the proposed bi-level resource allocation algorithm is that the first level resource allocation aims to guarantee the necessary condition in (6) by adjusting the minimum contention windows of both AP and background traffic users, and the second level resource allocation algorithm is performed by scheduling the video queues in AP properly to guarantee the fairness among the video users. Particularly, the bi-level resource allocation algorithm is invoked whenever AP obtains the opportunity to access the wireless channel.

The instantaneous required download rates of all the video users should be calculated carefully before each time when the bi-level resource allocation algorithm is performed, since the video bit rate is variable and the playback buffer is impacted by both the video bit rate and the download rate. In particular, if the video bit rate is larger than the download rate for a long duration, the playback buffer may go underflow and thus the playback will stall. Therefore, we try to maintain the playback buffer level (in seconds of playback) to a constant target $B_{\text {target }}$ and derive the corresponding instantaneous required download rates. Firstly, for each video user $i(1 \leq i \leq n)$, the average video bit rate $a_{i}$ obtained from the media present description (MPD) file is sent to AP before the start of the initial stage. Next, whenever video user $i$ receives a video data packet from AP, the buffer level parameter $B_{i}$ is fed back to AP by piggybacking it in the ACK packet. Then, when AP obtains the $k$-th ( $k$ is an arbitrary positive integer) opportunity to access the wireless channel, it calculates the current buffer level parameter $B_{i}^{k}$ for video user $i(1 \leq i \leq n)$. Especially, AP subtracts the time difference between the moment of receiving the latest ACK packet from video user $i$ and the current time from $B_{i}$ to obtain $B_{i}^{k}$. Eventually, based on the idea that the playback buffer should be filled to the level of $B_{\text {target }}$ before the end of duration $B_{i}^{k}$, the instantaneous required download rate $v_{i}^{k}$ is expressed as

$$
v_{i}^{k}=a_{i} \frac{B_{\text {target }}}{B_{i}^{k}}, \quad 1 \leq i \leq n .
$$

In the following, based on the instantaneous required download rates calculated above, we will detail the bi-level resource allocation algorithm, and then give the pseudo code of the algorithm.

\section{A. First level resource allocation algorithm}

The purpose of the first level resource allocation is to allocate enough wireless resource (downlink throughput) to AP to guarantee the sum of the required download rates of all the video users. To this end, we firstly tune the configuration of the network such that it will work at the optimal operation point where the maximum network throughput $S_{\max }$ can be achieved. Then the throughput of AP and background traffic users will be allocated according to their throughput requirements by properly assigning the minimum contention 
windows of them. According to our early studies [10], [13], the maximum network throughput $S_{\max }$ can be expressed as

$$
S_{\text {max }}=\frac{T_{l e n}}{T_{s}+\sigma K+T_{c}\left[K\left(e^{1 / K}-1\right)-1\right]},
$$

where $T_{s}$ and $T_{c}$ are the successful transmission time and the collision time, respectively. $T_{l e n}$ is the length of the packets in the network, $\sigma$ is the duration of an empty slot time, and $K=\sqrt{T_{c} / 2 \sigma}$.

According to (6), in order to guarantee the QoE of HTTP video streaming, the downlink throughput of $\mathrm{AP} r_{a p}^{k}$ (where the superscript $k$ represents that the variable is calculated when AP obtains the $k$-th opportunity to access the channel, similarly hereinafter) should be no less than the sum of instantaneous download rates of all the video users, therefore

$$
r_{a p}^{k}=\sum_{i=1}^{n} v_{i}^{k} .
$$

Furthermore, the residual throughput is allocated to all the background traffic users equally. Since all the background traffic users send packets to the AP, the number of background traffic users $m$ can be easily obtained by AP, then the throughput for each background traffic user is $r_{s t a}^{k}=\left(S_{\max }-r_{a p}^{k}\right) / m$. The ratio of the throughput of AP to that of background traffic user is

$$
\beta^{k}=r_{a p}^{k} / r_{s t a}^{k}=m \cdot r_{a p}^{k} /\left(S_{\max }-r_{a p}^{k}\right) .
$$

According to our earlier study [10], the new minimum contention windows for AP and background traffic users are calculated as follows

$$
\begin{gathered}
W_{a p}^{k}=\frac{2\left(1-2 p_{a p}^{k}\right)}{\left(1-2 p_{a p}^{k}\right) \tau_{a p}^{k}+p_{a p}^{k} \tau_{a p}^{k}\left[1-\left(2 p_{a p}^{k}\right)^{m_{-} a p}\right]}, \\
W_{\text {sta }}^{k}=\frac{2\left(1-2 p_{\text {sta }}^{k}\right)}{\left(1-2 p_{\text {sta }}^{k}\right) \tau_{\text {sta }}^{k}+p_{\text {sta }}^{k} \tau_{\text {sta }}^{k}\left[1-\left(2 p_{\text {sta }}^{k}\right)^{m_{-} \text {sta }}\right]},
\end{gathered}
$$

where the transmission probabilities of $\mathrm{AP}$ and background traffic users are $\tau_{a p}^{k}=1 /\left(K\left(1+\beta^{k} m\right)\right)$ and $\tau_{s t a}^{k}=$ $\beta^{k}\left(\tau_{a p}^{k} /\left(1-\tau_{a p}^{k}\right)\right) /\left(1+\beta^{k}\left(\tau_{a p}^{k}\left(1-\tau_{a p}^{k}\right)\right)\right)$ respectively. The collision probabilities of AP and background traffic users are $p_{a p}^{k}=1-\left(1-\tau_{\text {sta }}^{k}\right)^{m}$ and $p_{\text {sta }}^{k}=1-\left(1-\tau_{a p}^{k}\right)\left(1-\tau_{\text {sta }}^{k}\right)^{m-1}$ respectively.

\section{B. Second level resource allocation algorithm}

The second level resource allocation algorithm is performed by scheduling the video queues in AP according to their priorities, to adapt to the variable bit rate nature of video streaming and guarantee the fairness among the video users. A token-counter mechanism is adopted to calculate the priorities of the video queues. We define a token counter $N_{i}^{k}$ to denote the gap between the video data requirement and the video data actually obtained by video user $i$ when AP obtains the $k$-th channel access opportunity, which is calculated as

$$
N_{i}^{k}=\max \left(0, N_{i}^{k-1}+v_{i}^{k-1} \cdot \Delta k-C_{i}^{k-1}\right),
$$

where $v_{i}^{k-1}$ is the instantaneous required download rate of video user $i$ at the moment when AP obtains the $(k-1)$-th channel access opportunity, $\Delta k$ is the duration between then moments that AP obtains the $(k-1)$-th and $k$-th channel access opportunity, and $C_{i}^{k-1}$ is the amount of video data sent

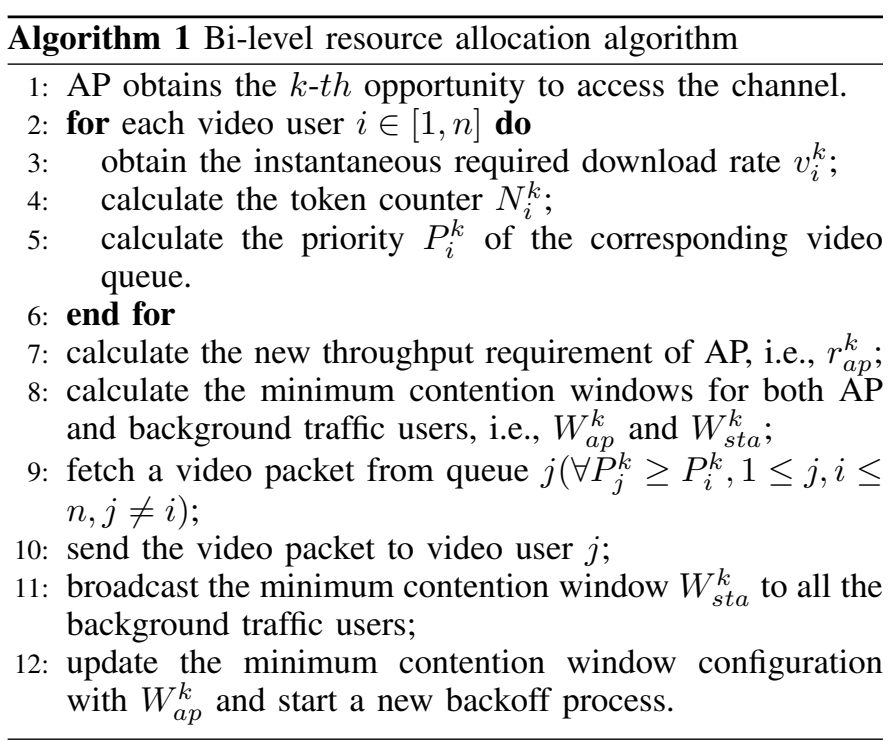

to video user $i$ at the moment when AP obtains the $(k-1)$-th channel access opportunity. $N_{i}^{k}$ reflects the service satisfaction of video user $i$, and the larger $N_{i}^{k}$, the more urgent the video user to be served. Meanwhile, the historical information, i.e., the average download rate for video user $i$, which is calculated by AP, should be also considered to calculate the priority. Therefore, the priority of queue $i$ is expressed as

$$
P_{i}^{k}=e^{\alpha_{i} N_{i}^{k}} \frac{1}{r_{i}^{k}}
$$

where $\alpha_{i}$ is a adjustable parameters larger than $0, r_{i}^{k}$ is the average download rate for video user $i$. The exponential form is used here because of it can response the fluctuation of token value quickly [14]. After the priorities of all the video queues are calculated, AP will choose the video queue with the highest priority to serve.

\section{Pseudo code}

The pseudo code of bi-level resource allocation algorithm is shown in Alg.1. The procedure in line 3 of Alg. 1 is used to update the basic parameters of the bi-level resource allocation algorithm, i.e., the instantaneous required download rates for all the video users. The priorities of video users are calculated by the procedures in lines 4 and 5 . Moreover, the main idea of the first level resource allocation algorithm is given in lines 7-8, and the main idea of the second level resource allocation algorithm is realized by the procedures in lines 9 and 10. Furthermore, the new contention window parameter for background traffic users $W_{\text {sta }}^{k}$ will broadcast to all the background traffic users, which will update their contention window with $W_{s t a}^{k}$ immediately after receiving this information. Finally, AP performs a new backoff process to contend for the next opportunity to access the channel by using the new $W_{a p}^{k}$.

\section{PERformance EVAluation}

\section{A. Simulation configuration}

In order to evaluate the performance of the proposed algorithm, extensive simulations using NS2 simulation tool [15] 
are conducted. We compare our bi-level resource allocation algorithm with IEEE 802.11e and IEEE 802.11 DCF with Round-Robin scheduling algorithm (hereinafter referred to as Round-Robin). The IEEE 802.11e protocol uses high priority access categories (AC2) to serve video traffic, but only provides one queue for the multiple video streaming and schedules the video packets with FIFO algorithm. IEEE 802.11 DCF uses the same access parameters for both AP and background traffic users. The Round-Robin is a simple and fairness scheduling algorithm in MAC layer and schedules the video queues in turn. The video traces used in the simulations are obtained from [16], which are encoded with H.264 standard. We choose the encoded quantization parameter as 48 , which means the video traces are encoded to very low average bit rates (24.095 to $31.993 \mathrm{Kbps})$. In the WLAN scenario, there are 15 video users, and each of which randomly selects one video file on the video server to download and playback. The number of background traffic users increases from 5 to 23 with a step of 2 . We set buffer level target $B_{\text {target }}$ as $5 \mathrm{~s}$ and the $\alpha_{i}(1 \leq i \leq n)$ in (14) as 0.01 . Other parameters about the network and video traces are shown in Table I.

The performance of the QoE is shown in Fig.2. It can be seen that the average playback fluency $F$ of our bi-level resource allocation algorithm is always very closed to 1 , which means the QoE of all the video users are guaranteed properly. However, the QoE performances of both IEEE 802.11e and Round-Robin are decreasing with the increasing number of background traffic users due to the ever-increasing collisions in the network. Since IEEE 802.11e assigns higher priority to AP as it serves video steaming, the QoE performance of IEEE $802.11 \mathrm{e}$ is better than that of Round-Robin.

\section{B. Simulation results}

Fig. 3 reveals the fairness among the video users versus the number of background traffic users. It is demonstrated that the standard derivation of $F$ of our algorithm is very small, which shows a great fairness. The fairness of IEEE 802.11e fluctuates with a high uncertainty and around a high average level, since IEEE 802.11e serves all the video streaming with only one queue and does not consider any difference between the video streaming. The fairness of Round-Robin decreases with the increasing number of background traffic users, which shows an increasing fairness. This can be explained by the fact that the playback fluency values of all the video streaming sessions of Round-Robin decrease in same proportion when the number of background traffic users increases due to the stationary resource allocation algorithm among the video users. However, only our bi-level resource allocation algorithm can guarantee the fairness all the time.

TABLE I. PARAMETERS

\begin{tabular}{l|l}
\hline Parameters & Value \\
\hline Video resolution & $1280 \times 720 \mathrm{p}$ \\
Video frame rate & $30 \mathrm{fps}$ \\
Video playback time & $600 \mathrm{~s}$ \\
MAC packet size & $1500 \mathrm{~B}$ \\
MAC control packet transmitting rate & $6 \mathrm{Mbps}$ \\
MAC data packet transmitting rate & $24 \mathrm{Mbps}$ \\
MAC RTS threshold & $2347 \mathrm{~B}$ \\
\hline
\end{tabular}

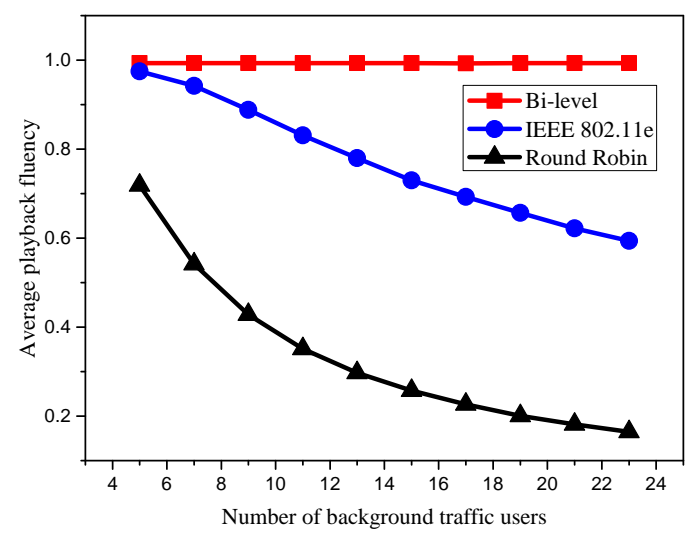

Fig. 2. QoE performance versus the number of background traffic users.

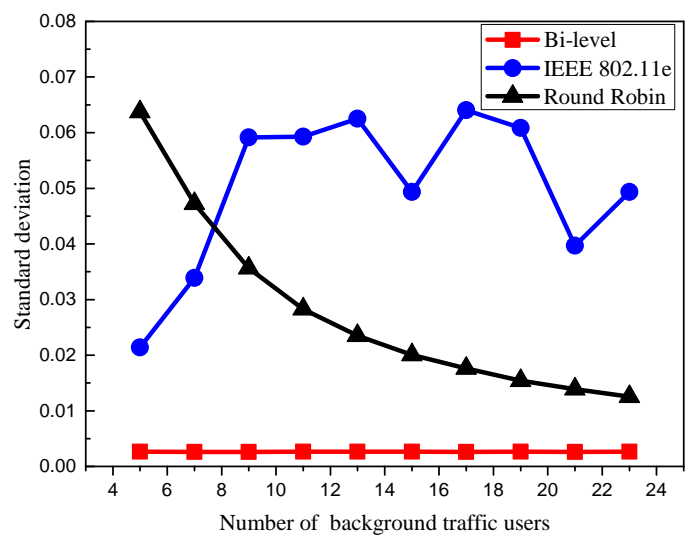

Fig. 3. Fairness versus the number of background traffic users.

Fig.4 shows the throughput of AP and average throughput of Background Traffic users (referred to as BT in the figure). The throughput of AP of our algorithm almost keeps a constant value since the bi-level resource allocation algorithm allocates throughput to AP mainly according to the instantaneous required download rates of the video users. The throughputs of AP of both IEEE 802.11e and Round-Robin decrease with the increasing of number of background traffic users because of the collisions in the network. IEEE 802.11e assigns higher priority to AP while Round-Robin serves both AP and backgrounds traffic users equally, therefore the throughput of AP of IEEE 802.11e is larger than that of Round-Robin. Furthermore, since we adjust the minimum contention windows dynamically to make the network working at the optimal operation point and the maximum throughput can be achieved, although our bilevel resource allocation allocates more resource to AP to guarantee the QoE of video users, the average throughput of background traffic users is no less than that of IEEE 802.11e.

\section{CONCLUSION}

The guarantee of QoE and fairness for multiple HTTP video streaming in WLAN is studied in this paper. By considering the instantaneous required download rates of video users over time, the first level resource allocation algorithm can adjust the throughput of AP to meet the download rate requirements of all the video users thus to avoid the stalling events, and the second level resource allocation algorithm can 


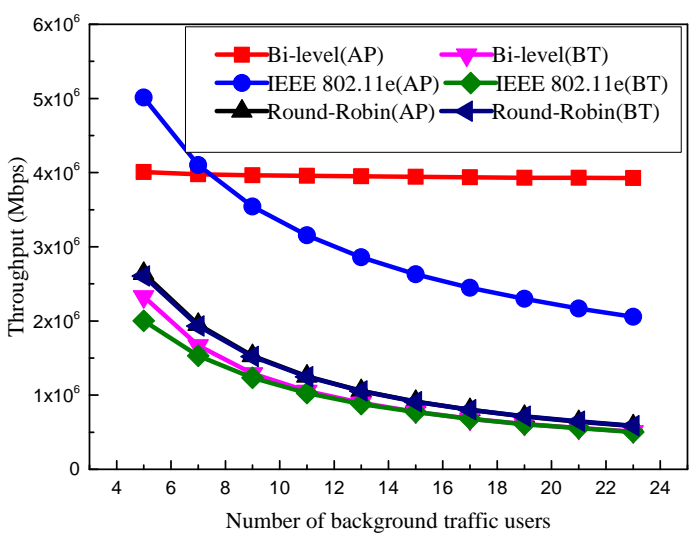

Fig. 4. Throughput of AP and the average throughput of background traffic users versus the number of background traffic users.

schedule the video queues in MAC layer to guarantee the fairness among video users. Simulation results show that both the QoE and fairness of multiple HTTP video streaming can be guaranteed properly and the impact of the average throughput of background traffic users is very little. In the future, the QoE performance of HTTP video streaming in next generation WLAN (e.g., IEEE 802.11ax) will be studied.

\section{ACKNOWLEDGMENT}

This work is supported in part by the National Natural Science Foundations of CHINA (Grant No. 61271279, and 61201157), the National 863 plans project (Grant No. 2014AA01A707, and 2015AA011307), the National Science and Technology Major Project (Grant No. 2015ZX03002006), and the Fundamental Research Funds for the Central Universities (Grant No. 3102015ZY038, 3102015ZY039).

\section{REFERENCES}

[1] Cisco, "Cisco visual networking index: Forecast and methodology,20132018," Cisco, Tech. Rep., 2014.

[2] C. Chen, L. K. Choi, G. de Veciana, C. Caramanis, R. Heath, and A. Bovik, "Modeling the time-varying subjective quality of http video streams with rate adaptations," Image Processing, IEEE Transactions on, vol. 23, no. 5, pp. 2206-2221, May 2014.

[3] Ericsson, "Ericsson mobility report in the pulse of the networked society," Report, Jun. 2014. [Online]. Available: http://www.ericsson.com/res/docs/2014/ericsson-mobility-reportjune-2014.pdf

[4] I.-T. SG12, "Definition of quality of experience," COM12 - LS 62 E, TD 109rev2 (PLEN/12), Geneva, Switzerland, Jan. 2007.

[5] R. Mok, E. Chan, and R. Chang, "Measuring the quality of experience of HTTP video streaming," in Integrated Network Management (IM), 2011 IFIP/IEEE International Symposium on, May. 2011, pp. 485-492.

[6] M. Seufert, S. Egger, M. Slanina, T. Zinner, T. Hobfeld, and P. TranGia, "A survey on quality of experience of http adaptive streaming," Communications Surveys Tutorials, IEEE, vol. 17, no. 1, pp. 469-492, Firstquarter 2015.

[7] N. Choi, J. Lee, and G. Kim, "A statistical approach to smooth video quality adaptation in ieee 802.11 wireless lans," in Information and Communication Technology Convergence (ICTC), 2014 International Conference on, Oct 2014, pp. 530-531.

[8] K. Miller, E. Quacchio, G. Gennari, and A. Wolisz, "Adaptation algorithm for adaptive streaming over http," in Packet Video Workshop (PV), 2012 19th International, May 2012, pp. 173-178.
[9] K. Miller, N. Corda, S. Argyropoulos, A. Raake, and A. Wolisz, "Optimal adaptation trajectories for block-request adaptive video streaming," in Packet Video Workshop (PV), 2013 20th International, Dec 2013, pp. $1-8$.

[10] H. Zhou, B. Li, M. Yang, and Z. Yan, "QoE-aware admission control and MAC layer parameter configuration algorithm in WLAN," wireless Communications and Networking Conference (WCNC), 2015 IEEE. In press.

[11] IEEE Standard for Information Technology-Telecommunications and Information Exchange Between Systems-Local and Metropolitan Area Networks-Specific Requirements Part 11: Wireless LAN Medium Access Control (MAC) and Physical Layer (PHY) Specifications Amendment 8: Medium Access Control (MAC) Quality of Service Enhancements, IEEE Amendment 802.11e, IEEE Std., 2005.

[12] IEEE Standard for Information Technology-Telecommunications and Information Exchange Between Systems-Local and Metropolitan Area Networks-Specific Requirements Part 11: Wireless LAN Medium Access Control (MAC) and Physical Layer (PHY) Specifications Amendment 2: MAC Enhancements for Robust Audio Video Streaming, IEEE Std.802.11aa-2012, IEEE Std., 2012.

[13] B. Li, R. Battiti, and Y. Fang, "Achieving optimal performance by using the IEEE 802.11 MAC protocol with service differentiation enhancements," Vehicular Technology, IEEE Transactions on, vol. 56, no. 3, pp. 1374-1387, May. 2007.

[14] A. L. Stolyar, "On the asymptotic optimality of the gradient scheduling algorithm for multiuser throughput allocation," Operations Research, vol. 53 , no. 1 , pp. $12-25,2005$.

[15] “Ns-2." [Online]. Available: www.isi.edu/nsnam/ns/.

[16] P. Seeling and M. Reisslein, "Video transport evaluation with h.264 video traces," vol. 14, no. 4, pp. 1142 - 1165, 2012. 